\title{
DIMENSION POLYNOMIALS AND THE EINSTEIN'S STRENGTH OF SOME SYSTEMS OF QUASI-LINEAR ALGEBRAIC DIFFERENCE EQUATIONS
}

\author{
ALEXANDER EVGRAFOV AND ALEXANDER LEVIN
}

\begin{abstract}
In this paper we present a method of characteristic sets for inversive difference polynomials and apply it to the analysis of systems of quasilinear algebraic difference equations. We describe characteristic sets and compute difference dimension polynomials associated with some such systems. Then we apply our results to the comparative analysis of difference schemes for some PDEs from the point of view of their Einstein's strength. In particular, we determine the Einstein's strength of standard finite-difference schemes for the Murray, Burgers and some other reaction-diffusion equations.
\end{abstract}

\section{INTRODUCTION}

Difference dimension polynomials, first introduced in [9] and [10, can be viewed as difference algebraic counterparts of Hilbert polynomials in commutative algebra and algebraic geometry, as well as of differential dimension polynomials in differential algebra. Difference dimension polynomials and their invariants are power tools for the study of difference and inversive difference field extensions, systems of algebraic difference equations, difference and inversive difference rings and modules (see, for example, [8, Ch. 6, 7], 9], [10, [11, [14, and [12, Sect. 3.6, 4.6]). Moreover, difference dimension polynomials play a significant role in the qualitative theory of difference equations, because the difference dimension polynomial of a system of algebraic difference equations expresses the Einstein's strength of the system (see [12, Chapter 7] for a detailed description of this concept).

In this paper we present a method of characteristic sets for inversive difference polynomials and apply it for the computation of difference dimension polynomials associated with difference schemes for some systems of quasi-linear algebraic PDEs. The characteristics sets of inversive difference polynomials were introduced in [8, Section 3.4]; their study was continued and extended to the case of several term orderings in [12, Section 2.3] and [13]. A similar theory for non-inversive difference polynomials was developed in [8, Section 3.4], [17, [5, 6] and some other works.

Hitherto, algorithmic methods for computing difference dimension polynomials (and therefore for determining the Einstein's strength) have been developed just for systems of linear difference equations. This work provides methods of computation of difference dimension polynomials for essentially wider class of systems of

Date: September 26, 2019.

2000 Mathematics Subject Classification. Primary 12H10; Secondary 39A10, 35K57.

Key words and phrases. Reaction-diffusion, Difference scheme, Difference polynomial, Characteristic set, Einstein's strength.

This work was completed with the support of the NSF grant CCF-1714425. 
difference equations. We prove the results on characteristic sets of difference ideals generated by quasi-linear difference polynomials that allow one to determine the Einstein's strength of important non-linear systems. In particular, we determine the strengths of systems of partial difference equations that arise from such schemes for reaction-diffusion PDEs. These equations play the key role in the theoretical foundation of the main methods for accurate, and rapid determination of biologically active organic carboxylic acids in objects such as infusion solutions and blood preservatives (see [4] for the description of the corresponding techniques).

\section{Preliminaries}

Throughout the paper, $\mathbb{N}, \mathbb{Z}, \mathbb{Q}$, and $\mathbb{R}$ denote the sets of all non-negative integers, integers, rational numbers, and real numbers, respectively. The number of elements of a set $A$ is denoted by Card $A$. As usual, $\mathbb{Q}[t]$ denotes the ring of polynomials in one variable $t$ with rational coefficients. All fields considered in the paper are supposed to be of characteristic zero. Every ring homomorphism is unitary (maps unity onto unity), every subring of a ring contains the unity of the ring.

If $B=A_{1} \times \cdots \times A_{k}$ is a Cartesian product of $k$ ordered sets with orders $\leq_{1}, \cdots \leq_{k}$, respectively $(k \in \mathbb{N}, k \geq 1)$, then by the product order on $B$ we mean a partial order $\leq_{P}$ such that $\left(a_{1}, \ldots, a_{k}\right) \leq_{P}\left(a_{1}^{\prime}, \ldots, a_{k}^{\prime}\right)$ if and only if $a_{i} \leq_{i} a_{i}^{\prime}$ for $i=1, \ldots, k$. In particular, if $a=\left(a_{1}, \ldots, a_{k}\right), a^{\prime}=\left(a_{1}^{\prime}, \ldots, a_{k}^{\prime}\right) \in \mathbb{N}^{k}$, then $a \leq_{P} a^{\prime}$ if and only if $a_{i} \leq a_{i}^{\prime}$ for $i=1, \ldots, k$. We write $a<_{P} a^{\prime}$ if $a \leq_{P} a^{\prime}$ and $a \neq a^{\prime}$. The lexicographic order on $\mathbb{N}^{k}$ is denoted by $\leq_{l e x}$. If it is strict, we use the symbol $<_{\text {lex }}$.

In this section we present some background material needed for the rest of the paper.

\subsection{Numerical polynomials of subsets of $\mathbb{N}^{m}$ and $\mathbb{Z}^{m}$.}

Definition 2.1. A polynomial $f(t)$ in one variable $t$ with rational coefficients is called numerical if $f(r) \in \mathbb{Z}$ for all sufficiently large $r \in \mathbb{Z}$.

Of course, every polynomial with integer coefficients is numerical. As an example of a numerical polynomial with non-integer coefficients one can consider a polynomial $\left(\begin{array}{l}t \\ k\end{array}\right)$ where $k \in \mathbb{N}$. (As usual, $\left(\begin{array}{l}t \\ k\end{array}\right)(k \geq 1$ ) denotes the polynomial $\frac{t(t-1) \ldots(t-k+1)}{k !},\left(\begin{array}{l}t \\ 0\end{array}\right)=1$, and $\left(\begin{array}{l}t \\ k\end{array}\right)=0$ if $k<0$.)

The following theorem proved in [7, Chapter 0, section 17] gives the "canonical" representation of a numerical polynomial.

Theorem 2.2. Let $f(t)$ be a numerical polynomial of degree $d$. Then $f(t)$ can be represented in the form

$$
f(t)=\sum_{i=0}^{d} a_{i}\left(\begin{array}{c}
t+i \\
i
\end{array}\right)
$$

with uniquely defined integer coefficients $a_{i}$.

In what follows (until the end of the section), we deal with subsets of $\mathbb{Z}^{m}$ ( $m$ is a positive integer). If $a=\left(a_{1}, \ldots, a_{m}\right) \in \mathbb{Z}^{m}$, then the number $\sum_{i=1}^{m}\left|a_{i}\right|$ will be 
called the order of the $m$-tuple $a$; it is denoted by ord $a$. Furthermore, the set $\mathbb{Z}^{m}$ will be considered as the union

$$
\mathbb{Z}^{m}=\bigcup_{1 \leq j \leq 2^{m}} \mathbb{Z}_{j}^{(m)}
$$

where $\mathbb{Z}_{1}^{(m)}, \ldots, \mathbb{Z}_{2^{m}}^{(m)}$ are all distinct Cartesian products of $m$ sets each of which is either $\mathbb{N}$ or $\mathbb{Z}_{-}=\{a \in \mathbb{Z} \mid a \leq 0\}$. We assume that $\mathbb{Z}_{1}^{(m)}=\mathbb{N}^{m}$ and call $\mathbb{Z}_{j}^{(m)}$ the $j$ th orthant of the set $\mathbb{Z}^{m}\left(1 \leq j \leq 2^{m}\right)$.

The set $\mathbb{Z}^{m}$ will be considered as a partially ordered set with the order $\unlhd$ defined as follows: $\left(e_{1}, \ldots, e_{m}\right) \unlhd\left(e_{1}^{\prime}, \ldots, e_{m}^{\prime}\right)$ if and only if the $m$-tuples $\left(e_{1}, \ldots, e_{m}\right)$ and $\left(e_{1}^{\prime}, \ldots, e_{m}^{\prime}\right)$ belong to the same orthant $\mathbb{Z}_{k}^{(m)}$ and the $m$-tuple $\left(\left|e_{1}\right|, \ldots,\left|e_{m}\right|\right)$ is less than $\left(\left|e_{1}^{\prime}\right|, \ldots,\left|e_{m}^{\prime}\right|\right)$ with respect to the product order on $\mathbb{N}^{m}$.

If $A \subseteq \mathbb{Z}^{m}$, then $W_{A}$ will denote the set of all elements of $\mathbb{Z}^{m}$ that do not exceed any element of $A$ with respect to the order $\unlhd$. (Thus, $w \in W_{A}$ if and only if there is no $a \in A$ such that $a \unlhd w$.) Furthermore, for any $r \in \mathbb{N}, A(r)$ will denote the set of all elements $x=\left(x_{1}, \ldots, x_{m}\right) \in A$ such that ord $x \leq r$.

The above notation can be naturally applied to subsets of $\mathbb{N}^{m}$ (treated as subsets of $\left.\mathbb{Z}^{m}\right)$. If $E \subseteq \mathbb{N}^{m}$ and $s \in \mathbb{N}$, then $E(s)$ will denote the set of all $m$-tuples $e=\left(e_{1}, \ldots, e_{m}\right) \in E$ such that ord $e \leq s$. Furthermore, we shall associate with a set $E \subseteq \mathbb{N}^{m}$ the set $V_{E} \subseteq \mathbb{N}^{m}$ that consists of all $m$-tuples $v=\left(v_{1}, \ldots, v_{m}\right) \in \mathbb{N}^{m}$ that are not greater than or equal to any $m$-tuple from $E$ with respect to the product order on $\mathbb{N}^{m}$. (Clearly, an element $v=\left(v_{1}, \ldots, v_{m}\right) \in \mathbb{N}^{m}$ belongs to $V_{E}$ if and only if for any element $\left(e_{1}, \ldots, e_{m}\right) \in E$, there exists $i \in \mathbb{N}, 1 \leq i \leq m$, such that $e_{i}>v_{i}$.)

The following two theorems proved, respectively, in [7, Chapter 0, section 17] and [8, Chapter 2] introduce certain numerical polynomials associated with subsets of $\mathbb{N}^{m}$ and give explicit formulas for the computation of these polynomials.

Theorem 2.3. Let $E$ be a subset of $\mathbb{N}^{m}$. Then there exists a numerical polynomial $\omega_{E}(t)$ with the following properties:

(i) $\omega_{E}(r)=$ Card $V_{E}(r)$ for all sufficiently large $r \in \mathbb{N}$.

(ii) $\operatorname{deg} \omega_{E}$ does not exceed $m$ and $\operatorname{deg} \omega_{E}=m$ if and only if $E=\emptyset$. In the last case, $\omega_{E}(t)=\left(\begin{array}{c}t+m \\ m\end{array}\right)$.

The polynomial $\omega_{E}(t)$ is called the dimension polynomial of the set $E \subseteq \mathbb{N}^{m}$.

Theorem 2.4. Let $E=\left\{e_{1}, \ldots, e_{q}\right\}(q \geq 1)$ be a finite subset of $\mathbb{N}^{m}$. Let $e_{i}=$ $\left(e_{i 1}, \ldots, e_{i m}\right) \quad(1 \leq i \leq q)$ and for any $l \in \mathbb{N}, 0 \leq l \leq q$, let $\Theta(l, q)$ denote the set of all $l$-element subsets of the set $\mathbb{N}_{q}=\{1, \ldots, q\}$. Furthermore, let $\bar{e}_{\emptyset_{j}}=0$ and for any $\theta \in \Theta(l, q), \theta \neq \emptyset$, let $\bar{e}_{\theta j}=\max \left\{e_{i j} \mid i \in \theta\right\}, 1 \leq j \leq m$. (In other words, if $\theta=\left\{i_{1}, \ldots, i_{l}\right\}$, then $\bar{e}_{\theta j}$ denotes the greatest $j$ th coordinate of the elements $\left.e_{i_{1}}, \ldots, e_{i_{l}}.\right)$ Furthermore, let $b_{\theta}=\sum_{j=1}^{m} \bar{e}_{\theta j}$. Then

$$
\omega_{E}(t)=\sum_{l=0}^{q}(-1)^{l} \sum_{\theta \in \Theta(l, q)}\left(\begin{array}{c}
t+m-b_{\theta} \\
m
\end{array}\right)
$$


Remark. Clearly, if $E \subseteq \mathbb{N}^{n}$ and $E^{*}$ is the set of all minimal elements of the set $E$ with respect to the product order on $\mathbb{N}^{m}$, then the set $E^{*}$ is finite and $\omega_{E}(t)=\omega_{E^{*}}(t)$. Thus, the last theorem gives an algorithm that allows one to find a numerical polynomial associated with any subset of $\mathbb{N}^{m}$ : one should first find the set of all minimal points of the subset and then apply Theorem 2.4.

The following two results, proved in [8, Section 2.5], describe dimension polynomials associated with subsets of $\mathbb{Z}^{m}$.

Theorem 2.5. Let $A$ be a subset of $\mathbb{Z}^{m}$. Then there exists a numerical polynomial $\phi_{A}(t)$ such that

(i) $\phi_{A}(r)=$ Card $W_{A}(r)$ for all sufficiently large $r \in \mathbb{N}$.

(ii) $\operatorname{deg} \phi_{A} \leq m$ and the polynomial $\phi_{A}(t)$ can be written in the form $\phi_{A}(t)=$ $\sum_{i=0}^{m} a_{i}\left(\begin{array}{c}t+i \\ i\end{array}\right)$ where $a_{i} \in \mathbb{Z}$ and $2^{m} \mid a_{m}$.

(iii) $\phi_{A}(t)=0$ if and only if $(0, \ldots, 0) \in A$.

(iv) If $A=\emptyset$, then $\phi_{A}(t)=\sum_{i=0}^{m}(-1)^{m-i} 2^{i}\left(\begin{array}{c}m \\ i\end{array}\right)\left(\begin{array}{c}t+i \\ i\end{array}\right)$.

Theorem 2.6. With the notation of Theorem 2.5, let us consider a mapping $\rho$ : $\mathbb{Z}^{m} \longrightarrow \mathbb{N}^{2 m}$ such that

$$
\rho\left(\left(e_{1}, \ldots, e_{m}\right)\right)=\left(\max \left\{e_{1}, 0\right\}, \ldots, \max \left\{e_{m}, 0\right\}, \max \left\{-e_{1}, 0\right\}, \ldots, \max \left\{-e_{m}, 0\right\}\right) .
$$

Let $B=\rho(A) \bigcup\left\{\bar{e}_{1}, \ldots, \bar{e}_{m}\right\}$ where $\bar{e}_{i}(1 \leq i \leq m)$ is a $2 m$-tuple in $\mathbb{N}^{2 m}$ whose $i$ th and $(m+i)$ th coordinates are equal to 1 and all other coordinates are equal to 0 . Then $\phi_{A}(t)=\omega_{B}(t)$ where $\omega_{B}(t)$ is the dimension polynomial of the set $B \subseteq \mathbb{N}^{2 m}$ (i. e., the dimension polynomial introduced in Theorem 2.3).

The polynomial $\phi_{A}(t)$ is called the dimension polynomial of the set $A \subseteq \mathbb{Z}^{m}$. It is easy to see that Theorems 2.6 and 2.4 provide an algorithm for computing such a polynomial.

2.2. Some basic facts from difference algebra. A difference ring is a commutative ring $R$ together with a finite set $\sigma=\left\{\alpha_{1}, \ldots, \alpha_{m}\right\}$ of mutually commuting injective endomorphisms of $R$ into itself. The set $\sigma$ is called the basic set of the difference ring $R$, and the endomorphisms $\alpha_{1}, \ldots, \alpha_{m}$ are called translations. A difference ring with a basic set $\sigma$ is also called a $\sigma$-ring. If $\alpha_{1}, \ldots, \alpha_{m}$ are automorphisms of $R$, we say that $R$ is an inversive difference ring with the basic set $\sigma$. In this case we denote the set $\left\{\alpha_{1}, \ldots, \alpha_{m}, \alpha_{1}^{-1}, \ldots, \alpha_{m}^{-1}\right\}$ by $\sigma^{*}$ and call $R$ a $\sigma^{*}$-ring. If a difference $(\sigma-)$ ring $R$ is a field, it is called a difference (or $\sigma_{-}$) field. If $R$ is inversive, it is called an inversive difference field or a $\sigma^{*}$-field. In what follows we deal only with inversive $\left(\sigma^{*}\right.$-) rings and fields where $\sigma=\left\{\alpha_{1}, \ldots, \alpha_{m}\right\}$.

If $R$ is a $\sigma^{*}$-ring and $R_{0}$ a subring of $R$ such that $\alpha\left(R_{0}\right) \subseteq R_{0}$ for any $\alpha \in \sigma^{*}$, then $R_{0}$ is called a $\sigma^{*}$-subring of $R$, while the ring $R$ is said to be a $\sigma^{*}$-overring of $R_{0}$. In this case the restriction of an endomorphism $\alpha_{i}$ on $R_{0}$ is denoted by the same symbol $\alpha_{i}$. If $R$ is a $\sigma^{*}$-field and $R_{0}$ a subfield of $R$, which is also a $\sigma^{*}$-subring of $R$, then $R_{0}$ is said to be a $\sigma^{*}$-subfield of $R ; R$, in turn, is called a $\sigma^{*}$-field extension or a $\sigma$-overfield of $R_{0}$. In this case we also say that we have a $\sigma^{*}$-field extension $R / R_{0}$. 
If $R$ is an inversive difference ring with a basic set $\sigma$ and $J$ is an ideal of $R$ such that $\alpha(J) \subseteq J$ for any $\alpha \in \sigma^{*}$, then $J$ is called a $\sigma^{*}$-ideal of $R$. If a $\sigma^{*}$-ideal $P$ of the ring $R$ is prime (in the usual sense), we say that $P$ is a prime $\sigma^{*}$-ideal of $R$. An element $a \in R$ is said to be a constant if $\alpha(a)=a$ for every $\alpha \in \sigma$.

If $R$ is a $\sigma^{*}$-ring, then $\Gamma$ will denote the free commutative group generated by the set $\sigma$. Elements of the group $\Gamma$ (written in the multiplicative form $\alpha_{1}^{i_{1}} \ldots \alpha_{m}^{i_{m}}$ with $i_{1}, \ldots, i_{m} \in \mathbb{Z}$ ) act on $R$ as automorphisms that are compositions of the automorphisms from the set $\sigma^{*}$.

If $S$ is a subset of a $\sigma^{*}$-ring $R$, then $[S]^{*}$ will denote the smallest $\sigma^{*}$-ideal of $R$ containing $S$; as an ideal, it is generated by the set $\Gamma S=\{\gamma(a) \mid \gamma \in \Gamma, a \in S\}$. If $S$ is finite, $S=\left\{a_{1}, \ldots, a_{k}\right\}$, we write $\left[a_{1}, \ldots, a_{k}\right]^{*}$ for $I=[S]^{*}$ and say that $I$ is a finitely generated $\sigma^{*}$-ideal of $R$. (In this case, elements $a_{1}, \ldots, a_{k}$ are said to be $\sigma^{*}$-generators of $I$.) If $R_{0}$ is a $\sigma^{*}$-subring of $R$, then the intersection of all $\sigma^{*}$-subrings of $R$ containing $R_{0}$ and a set $B \subseteq R$ is the smallest $\sigma^{*}$-subring of $R$ containing $R_{0}$ and $B$. This ring coincides with the ring $R_{0}[\{\gamma(b) \mid b \in B, \gamma \in \Gamma\}]$; it is denoted by $R_{0}\{B\}^{*}$. The set $B$ is said to be a set of $\sigma^{*}$-generators of $R_{0}\{B\}^{*}$ over $R_{0}$. If $B=\left\{b_{1}, \ldots, b_{k}\right\}$ is a finite set, we say that $R_{1}=R_{0}\{B\}^{*}$ is a finitely generated inversive difference (or $\sigma^{*}$-) ring extension (or overring) of $R_{0}$ and write $R_{1}=R_{0}\left\{b_{1}, \ldots, b_{k}\right\}^{*}$.

If $R$ is a $\sigma^{*}$-field, $R_{0}$ a $\sigma^{*}$-subfield of $R$ and $B \subseteq R$, then the intersection of all $\sigma^{*}$-subfields of $R$ containing $R_{0}$ and $B$ is denoted by $R_{0}\langle B\rangle^{*}$. This is the smallest $\sigma^{*}$-subfield of $R$ containing $R_{0}$ and $B$; it coincides with the field $R_{0}(\{\gamma(b) \mid b \in B, \gamma \in$ $\Gamma\})$. The set $B$ is called a set of $\sigma^{*}$-generators of the $\sigma^{*}$-field extension $R_{0}\langle B\rangle^{*}$ of $R_{0}$. If $B$ is finite, $B=\left\{b_{1}, \ldots, b_{k}\right\}$, we write $R_{0}\left\langle b_{1}, \ldots, b_{k}\right\rangle^{*}$ for $R_{0}\langle B\rangle^{*}$.

In what follows we often consider two or more inversive difference rings $R_{1}, \ldots, R_{p}$ with the same basic set $\sigma=\left\{\alpha_{1}, \ldots, \alpha_{m}\right\}$. Formally speaking, it means that for every $i=1, \ldots, p$, there is some fixed mapping $\nu_{i}$ from the set $\sigma$ into the set of all injective endomorphisms of the ring $R_{i}$ such that any two endomorphisms $\nu_{i}\left(\alpha_{j}\right)$ and $\nu_{i}\left(\alpha_{k}\right)$ of $R_{i}$ commute $(1 \leq j, k \leq n)$. We shall identify elements $\alpha_{j}$ with their images $\nu_{i}\left(\alpha_{j}\right)$ and say that elements of the set $\sigma$ act as mutually commuting automorphisms of the ring $R_{i}(i=1, \ldots, p)$.

Let $R_{1}$ and $R_{2}$ be inversive difference rings with the same basic set $\sigma=\left\{\alpha_{1}, \ldots, \alpha_{m}\right\}$. A ring homomorphism $\phi: R_{1} \rightarrow R_{2}$ is called a difference (or $\sigma-$ ) homomorphism if $\phi(\alpha(a))=\alpha(\phi(a))$ for any $\alpha \in \sigma, a \in R_{1}$. Clearly, if $\phi: R_{1} \rightarrow R_{2}$ is a $\sigma$ homomorphism of inversive difference rings, then $\phi\left(\alpha^{-1}(a)\right)=\alpha^{-1}(\phi(a))$ for any $\alpha \in \sigma, a \in R_{1}$. If a $\sigma$-homomorphism is an isomorphism (endomorphism, automorphism, etc.), it is called a difference (or $\sigma$-) isomorphism (respectively, difference (or $\sigma$-) endomorphism, difference (or $\sigma$-) automorphism, etc.). If $R_{1}$ and $R_{2}$ are two $\sigma^{*}$-overrings of the same $\sigma^{*}$-ring $R_{0}$ and $\phi: R_{1} \rightarrow R_{2}$ is a $\sigma$-homomorphism such that $\phi(a)=a$ for any $a \in R_{0}$, we say that $\phi$ is a difference (or $\sigma_{-}$) homomorphism over $R_{0}$ or that $\phi$ leaves the ring $R_{0}$ fixed.

It is easy to see that the kernel of any $\sigma$-homomorphism of $\sigma^{*}$-rings $\phi: R \rightarrow R^{\prime}$ is a $\sigma^{*}$-ideal of $R$. Conversely, let $g$ be a surjective homomorphism of a $\sigma^{*}$-ring $R$ onto a ring $S$ such that $\operatorname{Ker} g$ is a $\sigma^{*}$-ideal of $R$. Then there is a unique structure of a $\sigma^{*}$-ring on $S$ such that $g$ is a $\sigma$-homomorphism. In particular, if $I$ is a $\sigma^{*}$-ideal of a $\sigma^{*}$-ring $R$, then the factor ring $R / I$ has a unique structure of a $\sigma^{*}$-ring such that the canonical surjection $R \rightarrow R / I$ is a $\sigma$-homomorphism. In this case $R / I$ is said to be the $\sigma^{*}$-factor ring of $R$ by the $\sigma^{*}$-ideal $I$. 
If a $\sigma^{*}$-ring $R$ is an integral domain, then its quotient field $Q(R)$ can be naturally considered as a $\sigma^{*}$-overring of $R$. In this case $Q(R)$ is said to be the quotient $\sigma^{*}$ field of $R$. Clearly, if a $\sigma^{*}$-field $K$ contains $R$ as a $\sigma^{*}$-subring, then $K$ contains the quotient $\sigma^{*}$-field $Q(R)$.

Let $R$ be a $\sigma^{*}$-ring, $\Gamma$ the free commutative group generated by $\sigma$, and $Y=$ $\left\{y_{i} \mid i \in I\right\}$ a family of elements from some $\sigma^{*}$-overring of $R$. We say that the family $Y$ is transformally (or $\sigma$-algebraically) dependent over $R$, if the family $\Gamma(Y)=$ $\left\{\gamma\left(y_{i}\right) \mid i \in I, i \in I\right\}$ is algebraically dependent over $R$ (that is, there exist elements $v_{1}, \ldots, v_{k} \in \Gamma(Y)$ and a non-zero polynomial $f\left(X_{1}, \ldots, X_{k}\right)$ with coefficients in $R$ such that $\left.f\left(v_{1}, \ldots, v_{k}\right)=0\right)$. Otherwise, the family $Y$ is said to be transformally (or $\sigma$-algebraically) independent over $R$ or a family of inversive difference (or $\sigma^{*}$-) indeterminates over $R$. In the last case, the $\sigma^{*}$-ring $R\left\{\left(y_{i}\right)_{i \in I}\right\}^{*}$ is called the algebra of inversive difference (or $\sigma^{*}$-) polynomials over $R$. As it is shown in [8, Proposition 3.4.4], for any set $I$, there exists an algebra of $\sigma^{*}$-polynomials $S=R\left\{\left(y_{i}\right)_{i \in I}\right\}^{*}$ over $R$ in a family of $\sigma$-indeterminates $Y=\left\{y_{i} \mid i \in I\right\}$ with indices from the set $I$. If $S$ and $S^{\prime}$ are two such algebras, then there exists a $\sigma$-isomorphism $S \rightarrow S^{\prime}$ that leaves the ring $R$ fixed. If $R$ is an integral domain, then any algebra of $\sigma^{*}$-polynomials over $R$ is an integral domain.

The algebra of $\sigma^{*}$-polynomials in the family of $\sigma$-indeterminates $Y$ over $R$ can be constructed by extending the natural structure of a $\sigma^{*}$-ring from $R$ to the polynomial ring $S=R\left[\left\{y_{i, \gamma} \mid i \in I, \gamma \in \Gamma\right\}\right]$ in the set of indeterminates $\left\{y_{i, \gamma}\right\}$ indexed by $I \times \Gamma$. The extension of the action of an element $\beta \in \sigma^{*}$ from $R$ to $S$ is defined by $\beta\left(y_{i, \gamma}\right)=y_{i, \beta \gamma}(i \in I, \gamma \in \Gamma)$; in what follows, we denote $y_{i, 1}$ by $y_{i}$ and write $\gamma y_{i}$ for $y_{i, \gamma}$.

Remark 2.7. Power products of elements of $\sigma$ with nonnegative exponents form a commutative semigroup $T \subset \Gamma$; in the case of non-inversive difference rings, a family $Y=\left\{y_{i} \mid i \in I\right\}$ is said to be $\sigma$-algebraically independent over a $\sigma$-ring $R$ if the family $T(Y)=\left\{\tau y_{i} \mid \tau \in T, i \in I\right\}$ is algebraically independent over $R$. If $R$ is a $\sigma^{*}$-ring and $Y$ is a family of elements of some $\sigma^{*}$-overring of $R$, then the family $\Gamma(Y)$ is algebraically dependent over $R$ if and only if the family $T(Y)$ has this property. That is why we use the term " $\sigma$-algebraically dependent", not " $\sigma^{*}$-algebraically dependent".

Let $K$ be a $\sigma^{*}$-field and $L$ a $\sigma^{*}$-overfield of $K$. An element $u \in L$ is said to be transformally algebraic (or $\sigma$-algebraic) if the family $\{\gamma u \mid \gamma \in \Gamma\}$ is algebraic over $K$. Otherwise, we say that $u$ is transformally (or $\sigma$-) transcendental over $K$. As it is shown in [12, Sect. 4.1], there is a subset $B$ of $L$ such that $B$ is $\sigma$-algebraically independent over $K$ and every element of $L$ is $\sigma$-algebraic over $K\langle B\rangle^{*}$. Such a set $B$ is called a $\sigma$-transcendence basis of $L$ over $K$. All $\sigma$-transcendence bases of $L$ over $K$ have the same cardinality. If the $\sigma^{*}$-field extension $L / K$ is finitely generated, then every $\sigma$-transcendence basis of $L$ over $K$ is finite; the number of its elements is called the $\sigma$-transcendence degree of the extension $L / K$ and is denoted by $\sigma$-tr. $\operatorname{deg}_{K} L$.

If $K\left\{\left(y_{i}\right)_{i \in I}\right\}^{*}$ is an algebra of $\sigma^{*}$-polynomials over a $\sigma^{*}$-ring $K$ and $\left(\eta_{i}\right)_{i \in I}$ a family of elements from a $\sigma^{*}$-overfield of $K$, one can define a surjective $\sigma$ homomorphism $\phi_{\eta}: K\left\{\left(y_{i}\right)_{i \in I}\right\}^{*} \rightarrow K\left\{\left(\eta_{i}\right)_{i \in I}\right\}^{*}$ that maps every $y_{i}$ onto $\eta_{i}$ and leaves elements of $K$ fixed. This homomorphism is called the substitution of $\left(\eta_{i}\right)_{i \in I}$ for $\left(y_{i}\right)_{i \in I}$. If $g$ is a $\sigma^{*}$ - polynomial, then its image under a substitution of $\left(\eta_{i}\right)_{i \in I}$ for $\left(y_{i}\right)_{i \in I}$ is denoted by $g\left(\left(\eta_{i}\right)_{i \in I}\right)$. The kernel of $P$ of $\phi_{\eta}$ is a prime $\sigma^{*}$-ideal of 
$K\left\{\left(y_{i}\right)_{i \in I}\right\}^{*}$, since $K\left\{\left(\eta_{i}\right)_{i \in I}\right\}^{*}$ is an integral domain (it is contained in the field $L)$. Therefore, the $\sigma^{*}$-field $K\left\langle\left(\eta_{i}\right)_{i \in I}\right\rangle^{*}$ can be treated as the quotient $\sigma^{*}$-field of $R\left\{\left(y_{i}\right)_{i \in I}\right\}^{*} / P$.

Let $K$ be an inversive difference $\left(\sigma^{*}\right)$ field and $n$ a positive integer. By an $n$ tuple over $K$ we mean an $n$-dimensional vector $a=\left(a_{1}, \ldots, a_{n}\right)$ whose coordinates belong to some $\sigma^{*}$-overfield of $K$. If each $a_{i}(1 \leq i \leq n)$ is $\sigma$-algebraic over the $\sigma$-field $K$, we say that the $n$-tuple $a$ is $\sigma$-algebraic over $K$.

Let $R=K\left\{y_{1}, \ldots, y_{n}\right\}^{*}$ be the algebra of $\sigma^{*}$-polynomials in $n \sigma^{*}$-indeterminates $y_{1}, \ldots, y_{n}$ over $K$ and $\Phi=\left\{f_{j} \mid j \in J\right\} \subseteq R$. An $n$-tuple $\eta=\left(\eta_{1}, \ldots, \eta_{n}\right)$ over $K$ is said to be a solution of the set $\Phi$ or a solution of the system of algebraic difference equations $f_{j}\left(y_{1}, \ldots, y_{n}\right)=0(j \in J)$ if $\Phi$ is contained in the kernel of the substitution of $\left(\eta_{1}, \ldots, \eta_{n}\right)$ for $\left(y_{1}, \ldots, y_{n}\right)$. A system of algebraic difference equations $\Phi$ is said to be prime if the $\sigma^{*}$-ideal generated by $\Phi$ in the ring $R$ is prime.

Clearly, if one fixes an $n$-tuple $\eta=\left(\eta_{1}, \ldots, \eta_{n}\right)$ over a $\sigma^{*}$-field $K$, then all $\sigma^{*}$ polynomials of the ring $R=K\left\{y_{1}, \ldots, y_{n}\right\}^{*}$, for which $\eta$ is a solution, form a prime $\sigma^{*}$-ideal; it is called the defining $\sigma^{*}$-ideal of $\eta$ over $K$.

\section{Characteristic Sets and Difference Dimension Polynomials}

Let $K$ be an inversive difference $\left(\sigma^{*}\right)$ field with a basic set $\sigma=\left\{\alpha_{1}, \ldots, \alpha_{m}\right\}$ and let $\Gamma$ denote the free commutative group generated by $\sigma$. If $\gamma=\alpha_{1}^{k_{1}} \ldots \alpha_{m}^{k_{m}} \in \Gamma$ $\left(k_{1}, \ldots, k_{m} \in \mathbb{Z}\right)$, the order of the element $\gamma$ is defined as ord $\gamma=\sum_{i=1}^{m}\left|k_{i}\right|$. For any $r \in \mathbb{N}$, we set $\Gamma(r)=\{\gamma \in \Gamma \mid$ ord $\gamma \leq r\}$. Furthermore, for every $j=1, \ldots, 2^{m}$, we set $\Gamma_{j}=\left\{\gamma=\alpha_{1}^{k_{1}} \ldots \alpha_{m}^{k_{m}} \in \Gamma \mid\left(k_{1}, \ldots, k_{m}\right) \in \mathbb{Z}_{j}^{(m)}\right\}$ (see the representation (2.2) of the set $\mathbb{Z}^{m}$ as the union of the orthants).

Let $K\left\{y_{1}, \ldots, y_{n}\right\}^{*}$ be the algebra of $\sigma^{*}$-polynomials in $\sigma^{*}$-indeterminates $y_{1}, \ldots, y_{n}$ over $K$ and let $\Gamma Y$ denote the set $\left\{\gamma y_{i} \mid \gamma \in \Gamma, 1 \leq i \leq m\right\}$ whose elements are called terms. By the order of a term $u=\gamma y_{j}$ we mean the order of the element $\gamma \in \Gamma$. Furthermore, setting $(\Gamma Y)_{j}=\left\{\gamma y_{i} \mid \gamma \in \Gamma_{j}, 1 \leq i \leq 2_{2^{m}}\left(j=1, \ldots, 2^{m}\right)\right.$ we obtain a representation of the set of terms as a union $\Gamma Y=\bigcup_{j=1}^{2^{m}}(\Gamma Y)_{j}$.

Definition 3.1. A term $v \in \Gamma Y$ is called a transform of a term $u \in \Gamma Y$ if and only if $u$ and $v$ belong to the same set $(\Gamma Y)_{j}\left(1 \leq j \leq 2^{m}\right)$ and $v=\gamma u$ for some $\gamma \in \Gamma_{j}$. If $\gamma \neq 1, v$ is said to be a proper transform of $u$.

In what follows, we say that an element $\gamma \in \Gamma$ is similar to a term $u \in \Gamma Y$ and write $\gamma \sim u$ if $\gamma \in \Gamma_{j}$ and $u \in(\Gamma Y)_{j}$ for the same index $j\left(1 \leq j \leq 2^{m}\right)$. We also write $\gamma \sim \gamma^{\prime}$ if $\gamma, \gamma^{\prime} \in \Gamma_{j}$ and $u \sim v$ if $u, v \in(\Gamma Y)_{j}$ for the same $j$.

Definition 3.2. A well-ordering of the set of terms $\Gamma Y$ is called a ranking of the family of $\sigma^{*}$-indeterminates $y_{1}, \ldots, y_{n}$ (or a ranking of the set $\Gamma Y$ ) if it satisfies the following conditions. (We use the standard symbol $\leq$ for the ranking; it will be always clear what order is denoted by this symbol.)

(i) If $u \in(\Gamma Y)_{j}$ and $\gamma \in \Gamma_{j}\left(1 \leq j \leq 2^{m}\right)$, then $u \leq \gamma u$.

(ii) If $u, v \in(\Gamma Y)_{j}\left(1 \leq j \leq 2^{m}\right), u \leq v$ and $\gamma \in \Gamma_{j}$, then $\gamma u \leq \gamma v$. 
A ranking of the $\sigma^{*}$-indeterminates $y_{1}, \ldots, y_{n}$ is called orderly if for any $j=$ $1, \ldots, 2^{m}$ and for any two terms $u, v \in(\Gamma Y)_{j}$, the inequality ord $u<$ ord $v$ implies that $u<v$ (as usual, $v<w$ means $v \leq w$ and $v \neq w$ ).

As an example of an orderly ranking of the $\sigma^{*}$-indeterminates $y_{1}, \ldots, y_{n}$ one can consider the standard ranking defined as follows: $u=\alpha_{1}^{k_{1}} \ldots \alpha_{m}^{k_{m}} y_{i} \leq v=$ $\alpha_{1}^{l_{1}} \ldots \alpha_{m}^{l_{m}} y_{j}$ if and only if the $(2 m+2)$-tuple $\left(\sum_{\nu=1}^{m}\left|k_{\nu}\right|,\left|k_{1}\right|, \ldots,\left|k_{m}\right|, k_{1}, \ldots, k_{m}, i\right)$ is less than or equal to the $(2 m+2)$-tuple $\left(\sum_{\nu=1}^{m}\left|l_{\nu}\right|,\left|l_{1}\right|, \ldots,\left|l_{m}\right|, l_{1}, \ldots, l_{m}, j\right)$ with respect to the lexicographic order on $\mathbb{Z}^{2 m+2}$.

In what follows, we assume that an orderly ranking $\leq$ of the set of $\sigma^{*}$-indeterminates $y_{1}, \ldots, y_{n}$ is fixed. If $A \in K\left\{y_{1}, \ldots, y_{n}\right\}^{*}$, then the greatest (with respect to the ranking $\leq$ ) term that appears in $A$ is called the leader of $A$; it is denoted by $u_{A}$. If $u=u_{A}$ and $d=\operatorname{deg}_{u} A$, then the $\sigma^{*}$-polynomial $A$ can be written as $A=I_{d} u^{d}+I_{d-1} u^{d-1}+\cdots+I_{0}$ where $I_{k}(0 \leq k \leq d)$ do not contain $u$. The $\sigma^{*}$-polynomial $I_{d}$ is called the initial of $A$; it is denoted by $I_{A}$.

Definition 3.3. Let $A, B \in K\left\{y_{1} \ldots, y_{n}\right\}^{*}$. We say that $A$ has higher rank than $B$ and write $\operatorname{rk} A>\operatorname{rk} B$ if either $A \notin K, B \in K$, or $u_{A}$ has higher rank than $u_{B}$, or $u_{A}=u_{B}$ and $\operatorname{deg}_{u_{A}} A>\operatorname{deg}_{u_{A}} B$. If $u_{A}=u_{B}$ and $\operatorname{deg}_{u_{A}} A=\operatorname{deg}_{u_{A}} B$, we say that $A$ and $B$ have the same rank and write $\operatorname{rk} A=\operatorname{rk} B$.

Note that distinct $\sigma^{*}$-polynomials can have the same rank and if $A \notin K$, then $I_{A}$ has lower rank than $A$.

Definition 3.4. Let $A, B \in K\left\{y_{1}, \ldots, y_{n}\right\}^{*}$. The $\sigma^{*}$-polynomial $A$ is said to be reduced with respect to $B$ if $A$ does not contain any power of a transform $\gamma u_{B}$ $(\gamma \in \Gamma)$ whose exponent is greater than or equal to $\operatorname{deg}_{u_{B}} B$ (recall that by the definition of a transform, $\gamma \sim u_{B}$ ). If $\mathcal{A} \subseteq K\left\{y_{1}, \ldots, y_{n}\right\}^{*} \backslash K$, then a $\sigma^{*}$-polynomial $A \in K\left\{y_{1}, \ldots, y_{n}\right\}^{*}$, is said to be reduced with respect to $\mathcal{A}$ if $A$ is reduced with respect to every element of the set $\mathcal{A}$.

A set $\mathcal{A} \subseteq K\left\{y_{1}, \ldots, y_{n}\right\}^{*}$ is said to be autoreduced if either it is empty or $\mathcal{A} \cap K=\emptyset$ and every element of $\mathcal{A}$ is reduced with respect to all other elements of the set $\mathcal{A}$.

The proof of the following proposition can be obtained by mimicking the proof of the corresponding statement about autoreduced sets of differential polynomials, see [7, Ch. 1, Sect. 9].

Proposition 3.5. Every autoreduced set is finite and distinct elements of an autoreduced set have distinct leaders.

Theorem 3.6. (12, Theorem 2.4.7]) Let $\mathcal{A}=\left\{A_{1}, \ldots, A_{p}\right\}$ be an autoreduced subset in the ring of $\sigma^{*}$-polynomials $K\left\{y_{1}, \ldots, y_{n}\right\}^{*}$ and let $D \in K\left\{y_{1}, \ldots, y_{n}\right\}^{*}$. Furthermore, let $I(\mathcal{A})$ denote the set of all $\sigma^{*}$-polynomials $B \in K\left\{y_{1}, \ldots, y_{n}\right\}^{*}$ such that either $B=1$ or $B$ is a product of finitely many polynomials of the form $\gamma\left(I_{A_{i}}\right)$ where $\gamma \in \Gamma, i=1, \ldots, p$. Then there exist $\sigma^{*}$-polynomials $J \in I(\mathcal{A})$ and $D_{0} \in$ $K\left\{y_{1}, \ldots, y_{n}\right\}^{*}$ such that $D_{0}$ is reduced with respect to $\mathcal{A}$ and $J D \equiv D_{0}(\bmod [\mathcal{A}])$.

Note that, with the notation of the last theorem, the process of reduction that leads to the $\sigma^{*}$-polynomials $J \in I(\mathcal{A})$ and $D_{0}$ is algorithmic; the steps of the 
corresponding algorithm are similar to the steps described in the proof of Theorem 2.4.1 of [12]. The $\sigma^{*}$-polynomial $D_{0}$ is called the remainder of $D$ with respect to $\mathcal{A}$. We also say that $D$ reduces to $D_{0}$ modulo $\mathcal{A}$.

In what follows elements of an autoreduced set in $K\left\{y_{1}, \ldots, y_{n}\right\}^{*}$ will be always written in the order of increasing rank. With this assumption we introduce the following partial order on the set of all autoreduced sets.

Definition 3.7. Let $\mathcal{A}=\left\{A_{1}, \ldots, A_{p}\right\}$ and $\mathcal{B}=\left\{B_{1}, \ldots, B_{q}\right\}$ be two autoreduced sets of $\sigma^{*}$-polynomials in $K\left\{y_{1}, \ldots, y_{n}\right\}^{*}$. We say that $\mathcal{A}$ has lower rank than $\mathcal{B}$ and write $\operatorname{rk} \mathcal{A}<\operatorname{rk} \mathcal{B}$ if either there exists $k \in \mathbb{N}, 1 \leq k \leq \min \{p, q\}$, such that $\operatorname{rk} A_{i}=\operatorname{rk} B_{i}$ for $i=1, \ldots, k-1$ and $\operatorname{rk} A_{k}<\operatorname{rk} B_{k}$, or $p>q$ and $\operatorname{rk} A_{i}=\operatorname{rk} B_{i}$ for $i=1, \ldots, q$.

Mimicking the arguments of [7, Ch. 1, Sect. 9], one obtains that every nonempty family of autoreduced subsets of $K\left\{y_{1}, \ldots, y_{n}\right\}^{*}$ contains an autoreduced set of lowest rank. In particular, if $\emptyset \neq J \subseteq F\left\{y_{1}, \ldots, y_{n}\right\}^{*}$, then the set $J$ contains an autoreduced set of lowest rank called a characteristic set of $J$.

Proposition 3.8. (12, Proposition 2.4.8]) Let $K$ be an inversive difference field with a basic set $\sigma, J$ a $\sigma^{*}$-ideal of the algebra of $\sigma^{*}$-polynomials $K\left\{y_{1}, \ldots, y_{n}\right\}^{*}$, and $\mathcal{A}$ a characteristic set of $J$. Then

(i) The ideal $J$ does not contain nonzero $\sigma^{*}$-polynomials reduced with respect to $\mathcal{A}$. In particular, if $A \in \mathcal{A}$, then $I_{A} \notin J$.

(ii) If $J$ is a prime $\sigma^{*}$-ideal, then $J=[\mathcal{A}]^{*}: \Upsilon(\mathcal{A})$ where $\Upsilon(\mathcal{A})$ denotes the set of all finite products of elements of the form $\gamma\left(I_{A}\right)(\gamma \in \Gamma, A \in \mathcal{A})$.

A $\sigma^{*}$-ideal of the ring of $\sigma^{*}$-polynomials $K\left\{y_{1}, \ldots, y_{n}\right\}^{*}$ is called linear if it is generated (as a $\sigma^{*}$-ideal) by homogeneous linear $\sigma^{*}$-polynomials, i. e., $\sigma^{*}$-polynomials of the form $\sum_{i=1}^{p} a_{i} \gamma_{i} y_{k_{i}}\left(a_{i} \in K, \gamma_{i} \in \Gamma, 1 \leq k_{i} \leq n\right.$ for $\left.i=1, \ldots, p\right)$. As it is shown in [12, Proposition 2.4.9], every linear $\sigma^{*}$-ideal in $K\left\{y_{1}, \ldots, y_{n}\right\}^{*}$ is prime. A $\sigma^{*}$-polynomial is said to be quasi-linear if it is linear with respect to its leader.

Theorem 3.9. Let $K$ be an inversive difference field with a basic set $\sigma$ and let $\preccurlyeq$ be a preorder on $K\left\{y_{1}, \ldots, y_{n}\right\}^{*}$ such that $A_{1} \preccurlyeq A_{2}$ if and only if $u_{A_{2}}$ is a transform of $u_{A_{1}}$ and $\operatorname{deg}_{u_{A_{1}}} A_{1} \leq \operatorname{deg}_{u_{A_{2}}} A_{2}$. Furthermore, let $A$ be an irreducible $\sigma^{*}$-polynomial in $K\left\{y_{1}, \ldots, y_{n}\right\}^{*} \backslash K$ and $\Gamma A=\{\gamma A \mid \gamma \in \Gamma\}$. Then the set $\mathcal{M}$ of all minimal (with respect to $\preccurlyeq$ ) elements of $\Gamma A$ is a characteristic set of the $\sigma^{*}$-ideal $[A]^{*}$.

Proof. By [12, Theorem 2.4.13], if $M$ is a nonzero $\sigma^{*}$-polynomial in $[A]^{*}$ written as $M=\sum_{i=1}^{l} C_{i} A_{i} \quad(l \geq 1)$, where $C_{i} \in K\left\{y_{1}, \ldots, y_{n}\right\}^{*}(1 \leq i \leq l)$ and $A_{i}=\gamma_{i} A$ for some distinct elements $\gamma_{1}, \ldots, \gamma_{l} \in \Gamma$, then $\operatorname{deg}_{u_{A_{k}}} M \geq \operatorname{deg}_{u_{A_{k}}} A_{k}$ for some $k, 1 \leq k \leq l$. It follows that if $\gamma A$ is an element of $\mathcal{M}$ such that $\gamma A \preccurlyeq A_{k}$, then $M$ is not reduced with respect to $\gamma A$. Thus, the $\sigma^{*}$-ideal $[A]^{*}$ contains no nonzero $\sigma^{*}$-polynomial reduced with respect to $\mathcal{M}$, so $\mathcal{M}$ is a characteristic set of this ideal.

Proposition 3.10. Let $K$ be an inversive difference field with a basic set $\sigma=$ $\left\{\alpha_{1}, \ldots, \alpha_{m}\right\}, R=K\left\{y_{1}, \ldots, y_{n}\right\}^{*}$ the ring of $\sigma^{*}$-polynomials in $\sigma^{*}$-indeterminates 
$y_{1}, \ldots, y_{n}$ over $K$, and $A$ a quasi-linear (not necessarily irreducible) $\sigma^{*}$-polynomial in $R \backslash K$ with leader $u_{A}$. Furthermore, let $M$ be a nonzero $\sigma^{*}$-polynomial in the ideal $[A]^{*}$ of $R$ written in the form $M=\sum_{i=1}^{l} C_{i} A_{i} \quad(l \geq 1)$ where $C_{i} \in K\left\{y_{1}, \ldots, y_{n}\right\}^{*}$ $(1 \leq i \leq l)$ and $A_{i}=\gamma_{i} A$ for some distinct elements $\gamma_{1}, \ldots, \gamma_{l} \in \Gamma$ such that $\gamma_{i} \sim u_{A}$ for $i=1, \ldots, l$. Finally, let $u_{i}$ denote the leader of the $\sigma^{*}$-polynomial $A_{i}$ $(i=1, \ldots, l)$. Then there exists $\nu \in \mathbb{N}, 1 \leq \nu \leq l$, such that $\operatorname{deg}_{u_{\nu}} M \geq 1$.

Proof. Note that even though the quasi-linear $\sigma^{*}$-polynomial $A$ is not necessarily irreducible, it is irreducible as a polynomial in its leader $u_{A}$ over the field of rational functions in other terms of $A$. It follows that one can use the arguments of the proof of [12, Theorem 2.4.13]; this theorem assumes that $A$ is irreducible, but the proof actually uses only the fact that $A$ is irreducible as a univariate polynomial in $u_{A}$ whose coefficients are rational functions of the other terms of $A$. With this remark, our theorem becomes a consequence of [12, Theorem 2.4.13].

Proposition 3.11. With the notation of the last proposition, let $A$ be a quasilinear $\sigma^{*}$-polynomial in $K\left\{y_{1}, \ldots, y_{n}\right\}^{*} \backslash K$ of the form $A=a u_{A}+B$ where $a \in K$ and $B \in K\left\{y_{1}, \ldots, y_{n}\right\}^{*}$ (all terms of $B$ are smaller than $u_{A}$ ). Then the $\sigma^{*}$ ideal $[A]^{*}$ of $K\left\{y_{1}, \ldots, y_{n}\right\}^{*}$ is prime. Furthermore, the set $\mathcal{M}$ of all minimal (with respect to $\preccurlyeq$ ) elements of $\Gamma A$ (we use the notation of Theorem 3.9) is a characteristic set of $[A]^{*}$.

Proof. Without loss of generality we can assume that $a=1$. Let $u_{A} \in(\Gamma Y)_{j}$ $\left(1 \leq j \leq 2^{m}\right)$.

If $F E \in[A]^{*}(F, E \in R)$, then $F E$ can be written as $F E=\sum_{i=1}^{s} C_{i} \gamma_{i} A$ where $C_{i} \in R$ and $\gamma_{i} \in \Gamma(1 \leq i \leq s)$. Then one can take $\gamma \in \Gamma_{j}$ such that all terms of the $\sigma^{*}$-polynomials $\gamma F, \gamma G, \gamma C_{i}$ and $\gamma \gamma_{i} A(1 \leq i \leq s)$ lie in $(\Gamma Y)_{j}$. Applying $\gamma$ to the last equality we obtain that $(\gamma F)(\gamma E)=\sum_{i=1}^{s}\left(\gamma C_{i}\right)\left(\gamma \gamma_{i} A\right)$. Thus, without loss of generality we can assume that all terms of $F, E$ and $C_{i}(1 \leq i \leq s)$ in the representation $F E=\sum_{i=1}^{s} C_{i} \gamma_{i} A$ belong to $(\Gamma Y)_{j}$ and $\gamma_{i} \sim u_{A}$ for $i=1, \ldots, s$. Now one can subtract from $F$ some linear combination of elements of the form $\beta A$ with $\beta \in \Gamma_{j}$ to eliminate all transforms of $u_{A}$ in $F$. We obtain that $F \equiv$ $F_{1}\left(\bmod \left(\left\{\beta A \mid \beta \in \Gamma_{j}\right\}\right)\right)$ where $F_{1}$ does not contain any $\gamma u_{A}$ with $\gamma \in \Gamma_{j}$. Similarly, $E \equiv E_{1}\left(\bmod \left(\left\{\beta A \mid \beta \in \Gamma_{j}\right\}\right)\right)$ where $E_{1}$ does not contain any $\gamma u_{A}$ with $\gamma \in \Gamma_{j}$. If $F, E \notin[A]^{*}$, then $F_{1}, E_{1} \notin[A]^{*}$, but $F_{1} E_{1} \in[A]^{*}$, since $F E \in[A]^{*}$. At the same time, $F_{1} E_{1}$ does not contain any transform of $u_{A}$ and can be written as a linear combination of elements of the form $\beta A$ where $\beta \sim u_{A}$. We get a contradiction with the statement of Proposition 3.10, so our proposition is proved. (The last statement is a direct consequence of Theorem 3.9, since the $\sigma^{*}$-polynomial $A$ is irreducible.)

The following result, proved in [12, Theorem 4.2.5] introduces a Hilbert-type dimension polynomial associated with a prime $\sigma^{*}$-ideal of a ring of $\sigma^{*}$-polynomials.

Theorem 3.12. Let $K$ be an inversive difference field with a basic set of automorphisms $\sigma=\left\{\alpha_{1}, \ldots, \alpha_{m}\right\}, R=K\left\{y_{1}, \ldots, y_{n}\right\}^{*}$ the ring of $\sigma^{*}$-polynomials over $K$, 
and $P$ a prime $\sigma^{*}$-ideal of $R$. Let $L$ denote the quotient field of $R / P$ treated as the $\sigma^{*}$-field extension $K\left\langle\eta_{1}, \ldots, \eta_{n}\right\rangle^{*}$ of $K$ where $\eta_{i}(1 \leq i \leq n)$ is the canonical image of $y_{i}$ in $R / P$. Then there exists a polynomial $\psi_{\eta \mid K}(t) \in \mathbb{Q}[t]$ with the following properties.

(i) $\psi_{\eta \mid K}(r)=$ tr. $\operatorname{deg}_{K} K\left(\left\{\gamma \eta_{j} \mid \gamma \in \Gamma(r), 1 \leq j \leq n\right\}\right)$ for all sufficiently large $r \in \mathbb{N}$.

(ii) $\operatorname{deg} \psi_{\eta \mid K}(t) \leq m$ and the polynomial $\psi_{\eta \mid K}(t)$ can be written as

$$
\psi_{\eta \mid K}(t)=\frac{2^{m} a}{m !} t^{m}+o\left(t^{m}\right)
$$

where $a \in \mathbb{Z}$ and $o\left(t^{m}\right)$ is a polynomial of degree less than $m$.

(iii) The integers $a, d=\operatorname{deg} \psi_{\eta \mid K}(t)$ and the coefficient of $t^{d}$ in the polynomial $\psi_{\eta \mid K}(t)$ do not depend on the choice of a system of $\sigma$-generators $\eta$. Furthermore, $a=\sigma$-tr. $\operatorname{deg}_{K} L$.

(iv) Let $\mathcal{A}=\left\{A_{1}, \ldots, A_{p}\right\}$ be a characteristic set of the $\sigma^{*}$-ideal $P$ and for every $i=1, \ldots, n$, let

$$
E_{i}=\left\{\left(e_{i 1}, \ldots, e_{i m}\right) \in \mathbb{Z}^{m} \mid \alpha_{1}^{e_{i 1}} \ldots \alpha_{m}^{e_{i m}} y_{i} \text { is the leader of some element of } \mathcal{A}\right\}
$$

(of course, some sets $E_{i}$ might be empty). Then

$$
\psi_{\eta \mid K}(t)=\sum_{i=1}^{n} \phi_{E_{i}}(t)
$$

where $\phi_{E_{i}}(t)$ is the dimension polynomial of the set $E_{i} \subseteq \mathbb{Z}^{m}$ whose existence is established by Theorem 2.5.

The polynomial $\psi_{\eta \mid K}(t)$ whose existence is established by Theorem 3.12 is called the $\sigma^{*}$-dimension polynomial of the prime $\sigma^{*}$-ideal $P$. The last statement of Theorem 3.12, together with Theorems 2.6 and 2.4, gives a method of computation of the $\sigma^{*}$-dimension polynomial associated with a prime $\sigma^{*}$-ideal of the ring of $\sigma^{*}$ polynomials $K\left\{y_{1}, \ldots, y_{n}\right\}^{*}$. Therefore, it provides a method of computation of the Einstein's strength of a prime system of algebraic partial difference equations. (The $\sigma^{*}$-polynomials of such a system generate a prime $\sigma^{*}$-ideal $P$ of $K\left\{y_{1}, \ldots, y_{n}\right\}^{*}$; as it is explained in [12, Section 7.7], the Einstein's strength of the system is expressed by the $\sigma^{*}$-dimension polynomial of $P$.) In short, the $\sigma^{*}$-dimension polynomial determines the number of parameters in the general solution of the system that can be chosen arbitrarily (the "arbitrariness" of the general solution). Therefore, if two systems adequately describe a process, one should prefer to work with a system with the smaller $\sigma^{*}$-dimension polynomial. (Such polynomials are compared with respect to the natural order: $f(t) \leq g(t)$ if $f(r) \leq g(r)$ for all sufficiently large $r \in \mathbb{N}$.) In the next part of the paper, the results of Theorem 3.9, Proposition 3.11 and Theorem 3.12 will be used for the evaluation of the strength of systems of difference equations that represent finite-difference schemes for PDEs describing certain chemical processes.

\section{Evaluation of the Einstein's Strength of Difference SCHEMES For SOME REACTION-DIFFUSION EQUATIONS}

1. The diffusion equation in one spatial dimension for a constant collective diffusion coefficient $a$ and unknown function $u(x, t)$ describing the density of 
the diffusing material at given position $x$ and time $t$ is as follows:

$$
\frac{\partial u(x, t)}{\partial t}=c \frac{\partial^{2} u(x, t)}{\partial x^{2}}
$$

( $c$ is a constant). Let us compute the strength of difference equations that arise from three most common difference schemes for equation (4.1).

Strength of the forward difference scheme

The forward difference scheme for the diffusion equation (4.1) is based on the standard approximations $\frac{u(x+h, t)-u(x, t)}{h}$ and $\frac{u(x, t)-u(x, t+h)}{h}$ for $\frac{\partial u(x, t)}{\partial x}$ and $\frac{\partial u(x, t)}{\partial t}$, respectively, with a small step $h$.

We obtain the equation in finite differences

$$
u(x, t+h)-u(x, t)=a(u(x+2 h, t)-2 u(x+h, t)+u(x, t)) .
$$

where $a=c / h .\left(\frac{\partial^{2} u(x, t)}{\partial x^{2}}\right.$ is replaced with $\left.\frac{u(x+2 h, t)-2 u(x+h, t)+u(x, t)}{h^{2}}\right)$.

Let $K$ be an inversive difference functional field with basic set $\sigma=\left\{\alpha_{1}: f(x, t) \mapsto\right.$ $\left.f(x+h, t), \alpha_{2}: f(x, t) \mapsto f(x, t+h)\right\}(f(x, t) \in K)$ containing $a$ and let $K\{y\}^{*}$ be the ring of $\sigma^{*}$-polynomials in one $\sigma^{*}$-indeterminate $y$ over $K$. Treating $y$ as the unknown function $u(x, t)$ in the equation $(4.2)$, we can write this equation as

$$
a \alpha_{1}^{2} y-2 a \alpha_{1} y-\alpha_{2} y+(a+1) y=0 .
$$

Let $A$ denote the $\sigma^{*}$-polynomial in the left-hand side of the last equation. Since $A$ is linear, it generates a linear (and therefore a prime) $\sigma^{*}$-ideal $P=[A]^{*}$ in $K\{y\}^{*}$.

Applying Proposition 3.11, we obtain a characteristic set $\mathcal{A}=\left\{A_{1}, A_{2}, A_{3}, A_{4}\right\}$ of the ideal $P$ where

$$
\begin{array}{r}
A_{1}=A=a \alpha_{1}^{2} y-2 a \alpha_{1} y-\alpha_{2} y+(a+1) y, \\
A_{2}=\alpha_{1}^{-1} A=-\alpha_{1}^{-1} \alpha_{2} y+a \alpha_{1} y+(a+1) \alpha_{1}^{-1} y-2 a y \\
A_{3}=\alpha_{1}^{-1} \alpha_{2}^{-1} A=a \alpha_{1} \alpha_{2}^{-1} y+(a+1) \alpha_{1}^{-1} \alpha_{2}^{-1} y-\alpha_{1}^{-1} y-2 a \alpha_{2}^{-1} y \\
A_{4}=\alpha_{1}^{-2} \alpha_{2}^{-1} A=(a+1) \alpha_{1}^{-2} \alpha_{2}^{-1} y-\alpha_{1}^{-2} y-2 a \alpha_{1} \alpha_{2}^{-1} y+a \alpha_{2}^{-1} y
\end{array}
$$

The leaders of these $\sigma^{*}$-polynomials are $\alpha_{1}^{2} y, \alpha_{1}^{-1} \alpha_{2} y, \alpha_{1} \alpha_{2}^{-1} y$, and $\alpha_{1}^{-2} \alpha_{2}^{-1} y$, respectively (they are written first in the $\sigma^{*}$-polynomials $A_{i}$ above). Therefore, the $\sigma^{*}$-dimension polynomial of equation (4.3) is equal to the dimension polynomial of the subset

$$
E=\{(2,0),(-1,1),(1,-1),(-2,-1)\}
$$

of $\mathbb{Z}^{2}$. Applying the results of theorems 2.6 and 2.4 we obtain that the $\sigma^{*}$-dimension polynomial of equation (4.3) that expresses the Einstein's strength of the forward difference scheme for (4.1) is

$$
\psi_{\text {Forw }}(t)=5 t \text {. }
$$

Note that in this case

$V_{E}(r)=\left\{\left(1, y_{1}\right) \mid 0 \leq y_{1} \leq r-1\right\} \bigcup\left\{\left(0, y_{2}\right) \mid-r \leq y_{2} \leq r\right\} \bigcup\left\{\left(-1, y_{3}\right) \mid-(r-1) \leq y_{3} \leq 0\right\}$ $\bigcup\{(x, 0) \mid-r \leq x \leq-2\}$ for any $r \in \mathbb{N}\left(y_{1}, y_{2}, y_{3}, x \in \mathbb{Z}\right)$.

Strength of the symmetric difference scheme 
Consider the symmetric difference scheme for the diffusion equation (4.1) obtained by replacing the partial derivatives $\frac{\partial^{2} u(x, t)}{\partial x^{2}}$ and $\frac{\partial u(x, t)}{\partial t}$ with $\frac{u(x+h, t)-2 u(x, t)+u(x-h, t)}{h^{2}}$ and $\frac{u(x, t+h)-u(x, t-h)}{2 h}$, respectively. It leads to the equation in finite differ-

$$
u(x, t+h)-u(x, t-h)=a(u(x+h, t)-2 u(x, t)+u(x-h, t))
$$

where $a=2 c / h$. As in the case of the forward difference scheme, let $K$ be an inversive difference functional field with basic set $\sigma=\left\{\alpha_{1}: f(x, t) \mapsto f(x+h, t), \alpha_{2}\right.$ : $f(x, t) \mapsto f(x, t+h)\}(f(x, t) \in K)$ and let $K\{y\}^{*}$ be the ring of $\sigma^{*}$-polynomials in one $\sigma^{*}$-indeterminate $y$ over $K$ ( $y$ is treated as the unknown function $u(x, t)$; we also assume that $a \in K)$. Then the equation (4.4) can be written as

$$
a \alpha_{1} y+a \alpha_{1}^{-1} y-\alpha_{2} y+\alpha_{2}^{-1} y-2 a y=0 .
$$

By Proposition 3.11, the characteristic set of the $\sigma^{*}$-ideal generated by the $\sigma^{*}$ polynomial $B=a \alpha_{1} y+a \alpha_{1}^{-1} y-\alpha_{2} y+\alpha_{2}^{-1} y-2 a y$ is $\left\{B, \alpha_{1}^{-1} B\right\}$. The leaders of $B$ and $\alpha_{1}^{-1} B$ are $\alpha_{1} y$ and $\alpha_{1}^{-2} y$, respectively. Now Theorem 3.12 shows that the strength of the equation (4.5) is expressed by the dimension polynomial $\phi_{E}(t)$ of the set $E=\{(1,0),(-2,0)\} \subseteq \mathbb{Z}^{2}$ (see Theorem 2.5). By Theorem 2.6, this polynomial coincides with the dimension polynomial $\omega_{E^{\prime}}(t)$ of the set

$$
E^{\prime}=\{(1,0,0,0),(0,0,2,0),(1,0,1,0),(0,1,0,1)\} \subseteq \mathbb{N}^{4} .
$$

Applying formula (2.3) we obtain that the strength of the equation (4.5), which expresses the symmetric difference scheme for (4.1), is represented by the $\sigma^{*}$ dimension polynomial

$$
\psi_{\text {Symm }}(t)=4 t .
$$

\section{Strength of the Crank-Nicholson scheme}

The Crank-Nicholson scheme (see [2, Section 4]) applied to the diffusion equation with the above interpretation of the shifts of arguments as two automorphisms $\alpha_{1}$ and $\alpha_{2}$ gives the algebraic difference equation of the form

$$
\alpha_{1} \alpha_{2} y+a_{1} \alpha_{1}^{-1} \alpha_{2} y+a_{2} \alpha_{1} y+a_{3} \alpha_{2} y+a_{4} \alpha_{1}^{-1} y+a_{5}=0
$$

where $a_{i}(1 \leq i \leq 5)$ are constants. Applying Proposition 3.11, we obtain that the $\sigma^{*}$-polynomial $C=\alpha_{1} \alpha_{2} y+a_{1} \alpha_{1}^{-1} \alpha_{2} y+a_{2} \alpha_{1} y+a_{3} \alpha_{2} y+a_{4} \alpha_{1}^{-1} y+a_{5}$ in the left-hand side of the last equation generates a prime $\sigma^{*}$-ideal of $K\{y\}^{*}$ whose characteristic set consists of the $\sigma^{*}$-polynomials $C, \alpha_{1}^{-1} C, \alpha_{2}^{-1} C$, and $\alpha_{1}^{-1} \alpha_{2}^{-1} C$. Their leaders are $\alpha_{1} \alpha_{2} y, \alpha_{1}^{-2} \alpha_{2} y, \alpha_{1} \alpha_{2}^{-1} y$, and $\alpha_{1}^{-2} \alpha_{2}^{-1} y$, respectively. Applying theorems 2.6 and 2.4 to the set $\{(1,1),(-2,1),(1,-1),(-2,-1)\} \subseteq \mathbb{Z}^{2}$ we obtain that the strength of the equation (4.6) is expressed by the dimension polynomial

$$
\psi_{\text {Crank-Nickolson }}(t)=6 t-1 \text {. }
$$

Thus, the symmetric difference scheme for the diffusion equation has higher strength (that is, smaller dimension polynomial) than the forward difference scheme and the Crank-Nicholson scheme, so the symmetric scheme is the best among these three schemes from the point of view of the Einstein's strength.

\section{Murray, Fisher, Burger and some other quasi-linear reaction- diffusion equations.}


Proposition 3.11 allows us to compute the strength of reaction-diffusion equations of the form

$$
\frac{\partial u}{\partial t}-\frac{\partial^{2} u}{\partial x^{2}}=H\left(u, \frac{\partial u}{\partial x}\right)
$$

where $u=u(x, t)$ is a function of space and time variables $x$ and $t$, respectively, and $H\left(u, \frac{\partial u}{\partial x}\right)$ is a nonlinear function of $u$ and $\frac{\partial u}{\partial x}$. Such equations have recently attracted a lot of attention in the context of chemical kinetics, mathematical biology and turbulence. The following PDEs, that are particular cases of equation (4.7), are in the core of the corresponding mathematical models.

Murray equation ([1], equation (4)):

$$
\frac{\partial^{2} u}{\partial x^{2}}+\mu_{1} u \frac{\partial u}{\partial t}+\mu_{2} u-\mu_{3} u^{2}=0, \quad\left(\mu_{1}, \mu_{2}, \mu_{3} \text { are constants }\right) .
$$

Burgers equation ([16, Section 17.1, (17.3)]):

$$
\frac{\partial^{2} u}{\partial x^{2}}-u \frac{\partial u}{\partial x}-\frac{\partial u}{\partial t}=0 .
$$

Fisher equation $([16$, Section $17.1,(17.4)])$ :

$$
\frac{\partial^{2} u}{\partial x^{2}}-u \frac{\partial u}{\partial t}+u(1-u)=0
$$

Huxley equation ([16, Section 17.1, (17.5)]):

$$
\frac{\partial^{2} u}{\partial x^{2}}-u \frac{\partial u}{\partial t}-u(k-u)(u-1)=0, \quad k \neq 0 .
$$

Burgers-Huxley equation ([16, Section 17.1, (17.7)]):

$$
\frac{\partial^{2} u}{\partial x^{2}}+u \frac{\partial u}{\partial x}-\frac{\partial u}{\partial t}+u(k-u)(u-1)=0, \quad k \neq 0 .
$$

FitzHugh-Nagumo equation ([16, Section 17.1, (17.8)]):

$$
\frac{\partial^{2} u}{\partial x^{2}}+u \frac{\partial u}{\partial x}+u(1-u)(a-u)=0, \quad a \neq 0 .
$$

The last six equations are of the form

$$
\frac{\partial^{2} u}{\partial x^{2}}+(a u+b) \frac{\partial u}{\partial x}+c \frac{\partial u}{\partial t}+F(u)=0
$$

where $a, b, c$ are constants $(c \neq 0, a b \neq 0)$ and $F(u)$ is a polynomial in one variable $u$ with coefficients in the ground functional field $K$. Therefore, the forward difference scheme for equations (4.8) - (4.13) leads to algebraic difference equations of the form

$$
\alpha_{1}^{2} y+(a y+b-2) \alpha_{1} y+c \alpha_{2} y+G(y)=0 .
$$

(As before, we set $y=u$, denote the automorphisms of the ground field $f(x, t) \mapsto$ $f(x+1, t)$ and $f(x, t) \mapsto f(x, t+1)$ by $\alpha_{1}$ and $\alpha_{2}$, respectively, and write the monomials in the left-hand side of the equation in the decreasing order of their highest terms. We also set $G(y)=F(y)-a y^{2}-(b+c-1) y$.)

Applying Proposition 3.11 we obtain that the $\sigma^{*}$-polynomial $A=\alpha_{1}^{2} y+(a y+$ $b-2) \alpha_{1} y+c \alpha_{2} y+G(y)$ generates a prime $\sigma^{*}$-ideal of $K\{y\}^{*}\left(\sigma=\left\{\alpha_{1}, \alpha_{2}\right\}\right)$. As 
in the case of equation (4.3), we obtain that the characteristic set of the ideal $[A]^{*}$ consists of the $\sigma^{*}$-polynomials $A, \alpha_{1}^{-1} A, \alpha_{1}^{-1} \alpha_{2}^{-1} A$ and $\alpha_{1}^{-2} \alpha_{2}^{-1} A$ with leaders $\alpha_{1}^{2} y$, $\alpha_{1}^{-1} \alpha_{2} y, \alpha_{1} \alpha_{2}^{-1} y$ and $\alpha_{1}^{-2} \alpha_{2}^{-1} y$, respectively. Therefore (as in the case of equation (4.3) ) the $\sigma^{*}$-dimension polynomial that expresses the Einstein's strength of the forward difference scheme for each of the equations (4.8) - (4.13) is equal to the dimension polynomial of the set $\{(2,0),(-1,1),(1,-1),(-2,-1)\} \subseteq \mathbb{Z}^{2}$, that is,

$$
\psi_{\text {Forw }}(t)=5 t \text {. }
$$

The symmetric difference scheme for equation (4.15) (and therefore for each of the equations (4.8) - (4.13)) gives an algebraic difference equation of the form

$$
(a y+b+1) \alpha_{1} y+(1-a y-b) \alpha_{1}^{-1} y+c \alpha_{2} y-c \alpha_{2}^{-1} y+F(y)=0 .
$$

(Recall that we replace $\frac{\partial u}{\partial x}, \frac{\partial^{2} u}{\partial x^{2}}$ and $\frac{\partial u}{\partial t}$ with $\left(\alpha_{1}+\alpha_{1}^{-1}-2\right) u,\left(\alpha_{1}-\alpha_{1}^{-1}\right) u$ and $\left(\alpha_{2}-\alpha_{2}^{-1}\right) u$, respectively.) If we consider the standard ranking defined after Definition 3.2, then the quasi-linear $\sigma^{*}$-polynomial in the left-hand side of the equation (4.16) is not of the form considered in Proposition 3.11. However, if one considers a similar ranking with $\alpha_{2}>\alpha_{1}$, then the $\sigma^{*}$-polynomial $B=$ $(a y+b+1) \alpha_{1} y+(1-a y-b) \alpha_{1}^{-1} y+c \alpha_{2} y-c \alpha_{2}^{-1} y+F(y)$ in the left-hand side of (4.16) is a quasi-linear one with the leader $\alpha_{2} y$. By Proposition 3.11, the $\sigma^{*}$ polynomials $B$ and $\alpha_{2}^{-1} B$ form a characteristic set of the prime $\sigma^{*}$-ideal $[B]^{*}$ of $K\{y\}^{*}$. Since their leaders are, respectively, $\alpha_{2} y$ and $\alpha_{2}^{-2} y$, the Einstein's strength of the symmetric difference scheme for each of the equations (4.8) - (4.13) is expressed by the dimension polynomial $\psi_{\text {Symm }}(t)$ of the set $\{(1,0),(0,2)\} \subseteq \mathbb{Z}^{2}$. As in the case of equation (4.5), we obtain that

$$
\psi_{\text {Symm }}(t)=4 t .
$$

Thus, one should prefer the symmetric scheme to the forward one while considering the Einstein's strength of these schemes for PDEs (4.8) - (4.13).

3. The mathematical model of chemical reaction kinetics with the diffusion phenomena is described by a system of partial differential equations of the form

$$
\left\{\begin{aligned}
\frac{\partial u_{1}}{\partial t} & =\frac{\partial^{2} u_{1}}{\partial x^{2}} \\
\frac{\partial u_{2}}{\partial t} & =\frac{\partial^{2} u_{2}}{\partial x^{2}} \\
\frac{\partial u_{1}}{\partial t} & =\frac{\partial^{2} u_{3}}{\partial x^{2}}-k_{1} u_{3}^{2}+k_{1} u_{3} u_{1}+k_{2} u_{2}-k_{2} u_{3}
\end{aligned}\right.
$$

(see [15]).

The forward difference scheme leads to the following system of algebraic difference equations with three $\sigma^{*}$-indeterminates $y_{1}, y_{2}$ and $y_{3}$ (they stand for $u_{1}, u_{2}$ and $u_{3}$, respectively), where $\sigma=\left\{\alpha_{1}: f(x, t) \mapsto f(x+1, t), \alpha_{2}: f(x, t) \mapsto f(x, t+1)\right\}$ $(f(x, t)$ is an element of the inversive ground functional field $K)$. 


$$
\left\{\begin{array}{l}
\alpha_{1}^{2} y_{1}-2 \alpha_{1} y_{1}-\alpha_{2} y_{1}+2 y_{1}=0 \\
\alpha_{1}^{2} y_{2}-2 \alpha_{1} y_{2}-\alpha_{2} y_{2}+2 y_{2}=0 \\
\alpha_{1}^{2} y_{3}-2 \alpha_{1} y_{3}-\alpha_{2} y_{3}+k_{1} y_{1} y_{3}-k_{1} y_{3}^{2}+k_{2} y_{2}-k_{2} y_{3}=0
\end{array}\right.
$$

where $k_{1}, k_{2}, k_{3}$ are constants in $K$.

Let $A_{1}, A_{2}$, and $A_{3}$ be the $\sigma^{*}$-polynomials in the left-hand sides of the first, second and third equations of the last system, respectively. Combining Proposition 2.4 .9 of [12] (that states that every linear $\sigma^{*}$-ideal in a ring of $\sigma^{*}$-polynomials is prime) and our Proposition 3.11 we obtain that the $\sigma^{*}$-ideal $\left[A_{1}, A_{2}, A_{3}\right]^{*}$ of the ring $K\left\{y_{1}, y_{2}, y_{3}\right\}^{*}$ is prime. Since $A_{1}$ and $A_{2}$ are linear $\sigma^{*}$-polynomials in different $\sigma^{*}$ indeterminates ( $y_{1}$ and $y_{2}$, respectively) and $A_{3}$ is a quasi-linear $\sigma^{*}$-polynomial with coefficient 1 of its leader $\alpha_{1}^{2} y_{3}$ (it can be treated as a quasi-linear $\sigma^{*}$-polynomial in $y_{3}$ over the quotient $\sigma^{*}$-field of $\left.K\left\{y_{1}, y_{2}, y_{3}\right\}^{*} /\left[A_{1}, A_{2}\right]^{*}\right)$, one can apply Proposition 3.11 to obtain that the twelve $\sigma^{*}$-polynomials $A_{i}, \alpha_{1}^{-1} A_{i}, \alpha_{1}^{-1} \alpha_{2}^{-1} A_{i}, \alpha_{1}^{-2} \alpha_{2}^{-1} A_{i}$ $(i=1,2,3)$ form a characteristic set of $\left[A_{1}, A_{2}, A_{3}\right]^{*}$ (cf. the characteristic set of the $\sigma^{*}$-ideal generated by the left-hand side of equation (4.3)). Proceeding as in the case of forward difference scheme of the diffusion equation we obtain that the strength of the forward difference scheme for system (4.17) is expressed by the polynomial

$$
\psi_{\text {Forw }}(t)=15 t \text {. }
$$

Using the above arguments and the results for difference schemes for equation (4.1), we obtain that the strengths of the symmetric and Crank-Nicholson schemes for (4.17) are expressed with the polynomials

$$
\psi_{\text {Symm }}(t)=12 t \text { and } \psi_{\text {Crank-Nicholson }}(t)=18 t-3,
$$

respectively. Therefore, in our case, as in the case of equation (4.1), the symmetric scheme for system (4.17) is characterized by the smallest $\sigma^{*}$-dimension polynomial (and therefore by the highest Einstein's strength) among these schemes.

\section{REFERENCES}

[1] Cherniha, R. M. New Ansätze and Exact Solutions for Nonlinear Reaction-Diffusion EquationsArising in Mathematical Biology. Symmetry in Nonlinear Mathematical Physics, 1 (1997), $138-146$.

[2] Crank, J.; Nicholson, P. A practical method for numerical evaluation of solutions of partial differential equations of the heat-conduction type. Advances in Computational Mathematics, 6 (I996) 207-226.

[3] Einstein, A. The Meaning of Relativity. Appendix II (Generalization of gravitation theory), 4th ed. Princeton, 133-165.

[4] Evgrafov, A. A. Standardization and Control of the Quality of Transfusion Liquids. Ph. D. Thesis. Sechenov First Moscow State Medical University, 1998.

[5] Gao, X. S.; Luo, Y.; C. Yuan, C. A Characteristic Set Method for Ordinary Difference Polynomial Systems. Journal of Symbolic Computation, 44 (2009), no. 3, 242-260.

[6] X.S. Gao, C. Yuan, and G. Zhang, Ritt-Wu's Characteristic Set Method for Ordinary Difference Polynomial Systems with Arbitrary Ordering. Acta Mathematica Scientia, 29 (2009), no. 3-4, 1063-1080.

[7] Kolchin, E. R. Differential Algebra and Algebraic Groups. Acad. Press, 1973.

[8] Kondrateva, M. V.; Levin, A. B.; Mikhalev, A. V.; Pankratev, E. V. Differential and Difference Dimension Polynomials. Kluwer Acad. Publ., 1998. 
[9] Levin, A. B. Characteristic polynomials of filtered difference modules and of difference field extensions. Russian Math. Surveys, 33 (1978), no.3, 165-166.

[10] Levin, A. B. Characteristic polynomials of inversive difference modules and some properties of inversive difference dimension. Russian Math. Surveys, 35 (1980), no. 1, 217-218.

[11] Levin, A. B. Type and dimension of inversive difference vector spaces and difference algebras. VINITI (Moscow, Russia), 1982, no. 1606-82, pp. 1-36.

[12] Levin, A. B. Difference Algebra. Springer, 2008.

[13] Levin, A. B. Multivariate Dimension Polynomials of Inversive Difference Field Extensions. Lecture Notes in Comput. Sci., 8372 (2014), 146-163.

[14] Levin, A. B.; Mikhalev, A. V. Type and Dimension of Finitely Generated G-algebras. Contemp. Math., 184 (1995), 275-280.

[15] Lim, J. Stability of solutions to a reaction diffusion system based upon chemical reaction kinetics. J. Math. Chem., 43 (2008), no. 3, 1134-1140.

[16] Wazwaz,A. M. Partial Differential Equations and Solitary Waves Theory. Springer, 2009.

[17] G.L. Zhang, G. L.; Gao, X. S. Properties of Ascending Chians fr Partial Difference Polynomial Systems. ASCM 2007: Computer Mathematics. LNAI 5081, 307-321, Springer-Verlag, Berlin Heidelberg, 2008.

Department of Analytical, Physical and Colloid Chemistry, Sechenov First Moscow State Medical University, Moscow 119991, Russia

E-mail address: afkx_farm@mail.ru

Department of Mathematics, The Catholic University of America, Washington, D.C. 20064

E-mail address: levin@cua.edu 